Y. C. Minh and E. F. van Dishoeck, eds.

\title{
Physical Processes Responsible for the Removal of Circumstellar Disks
}

\author{
Doug Johnstone \\ Dept. of Astronomy, University of Toronto, Ontario, Canada
}

\begin{abstract}
The most likely processes responsible for the removal of circumstellar disks around young stars are reviewed with emphasis on the physical state of the disk during the period of destruction and the timescale for disk removal. Four disk dispersal mechanisms are discussed in detail: 1) viscous accretion of material onto the central source, 2) close stellar encounters, 3) stellar winds, and 4) photoevaporation by ultraviolet radiation. While viscous accretion is shown to be efficient in the inner regions of disks $(r<10 \mathrm{AU})$, photoevaporation is the principal process of disk dispersal at large radii $(r>10 \mathrm{AU})$. The commonly held view that stellar winds removed the remnant Solar Nebula is seriously questioned.
\end{abstract}

\section{Introduction}

The disk environment in which the initial physical and chemical processing of pre-planetary materials is undertaken should not be represented as a simple, static structure but rather as a complex, dynamically evolving system. In some circumstellar environments the evolutionary time of the disk may approach or become shorter than the time required for agglomeration and coagulation of solids and gas into planets. Thus, while observations of planets around other stars have now been confirmed (Marcy, Cochran, \& Mayor 1999) there may be large classes of stars for which planetary systems are unexpected.

In this paper I will briefly review the important timescales required for the formation of terrestrial and gaseous planets according to present planet formation theories (§2). I will then discuss the various mechanisms responsible for the removal of circumstellar disks: viscosity in the disk results in the migration and accretion of disk materials $(\S 3)$; close encounters with nearby stars may produce tidal torques upon the disk, liberating disk material and planets ( $\S 4)$; stellar winds, especially the enhanced winds observed around young stars, may erode the disk through the entrainment of surface material along the thin boundary layer between the disk and wind $(\S 5)$; ultraviolet radiation, produced either locally by the central star or externally by nearby massive stars, is capable of heating the surface layer of the disk and powering pressure driven, thermal disk winds $(\S 6)$. A more detailed discussion of these disk removal processes may be found in the recent extended review by Hollenbach, Yorke, \& Johnstone (1999). 


\section{Planet Formation Timescales}

Standard star formation theories begin with a rotating cloud of gas which collapses to form a star and centrifically supported circumstellar disk (Terebey, Shu, \& Cassen 1984). For most stars, the majority of the infalling cloud material lands initially on the disk and must be accreted through the disk onto the star. When the disk mass becomes $\sim 0.3 \mathrm{M}_{*}$, where $\mathrm{M}_{*}$ is the mass of the protostar, the disk becomes gravitationally unstable, producing spiral density waves, and efficiently transporting disk material onto the protostar (Laughlin \& Bodenheimer 1994; Yorke, Bodenheimer, \& Laughlin 1995). Thus, it is reasonable to expect that at the end of the cloud accretion phase a typical star is surrounded by a disk accounting for around one quarter of the mass of the system (i.e. $1 \mathrm{M}_{\odot}$ star with a $0.3 \mathrm{M}_{\odot}$ circumstellar disk). Direct observational measurements of disk masses are difficult; however, in general the inferred mass of the disk is approximately $1 \%$ of the mass of the central star (Adams, Emerson, \& Fuller 1990; Beckwith et al. 1990). Either significant disk evolution occurs soon after cloud accretion ends or disk accretion onto the central star is much more efficient than anticipated during the star formation process.

Models for planet formation depend on converting dust and ice particles from atomic scales to planetary sizes. Even assuming that collisions between particles will result in total coalescence, extensive time is required to bring the individual constituents together. Gravitational focusing enhances interactions and leads to runaway growth of the most massive bodies (Safronov 1969; Wetherill 1980; Nakagawa et al. 1983) while the regular orbital motion of particles within the circumstellar disk tends to isolate bodies once they have consumed everything within their 'feeding-zone.' The current recipe for the formation of a giant planet requires the rapid accumulation of all solid (comet-like) bodies within a feeding-zone via runaway growth producing giant planet embryos with $M \sim 1$ $\mathrm{M}_{\oplus}$ within $\sim 10^{5} \mathrm{yr}$ (Weidenschilling 1984; Greenberg et al. 1978, 1984). Once isolated, a much longer time is required to gravitationally perturb the individual embryo orbits and produce collisions among the embryos (Wetherill \& Stewart 1986). At a critical core mass of $15-20 \mathrm{M}_{\oplus}$ the proto-giant planet begins to accumulate gas much faster than it gathers solids (Mizuno 1980; Bodenheimer \& Pollack 1986; Pollack et al. 1996), a process which concludes only when the gas is removed from the disk or the tidal torques produced by the planet are sufficient to open a gap around the planet in the gaseous disk (Papaloizou \& Lin 1984; Lin \& Papaloizou 1985, 1986a,b).

The strongest constraint on the timescale for formation of giant planets is the time required to mutually perturb the planet embryos into crossing orbits and produce collisions resulting in massive proto-giant planet cores. If the disk has a low mass, similar to the 'minimum mass' required to form the planets in our solar system $M_{d} \sim 0.01 \mathrm{M}_{\odot}$ (Hayashi et al. 1985), the time required to assemble the giant planets ranges from $10^{8} \mathrm{yr}$ for Jupiter to $10^{11} \mathrm{yr}$ for Neptune (Nakagawa et al. 1983), much too long to explain the solar system. Increasing the mass of the circumstellar disk from which the planets formed alleviates this problem. If the initial disk mass was $M_{d} \sim 0.1 \mathrm{M}_{\odot}$ then according to Lissauer $(1987,1993)$ the slow process of embryo collisions can be removed and core growth of the giant planets occurs on timescales of $10^{6}-10^{7} \mathrm{yr}$. 


\section{Viscous Accretion Disks}

Within the circumstellar disk an inward flow of material must be offset by an outward transportation of angular momentum via turbulence or magnetic fields. Thus, as the circumstellar disk accretes matter onto the central star it also spreads outwards lowering the accretion rate. If the total angular momentum of the disk $J_{d}$ is fixed, the characteristic disk radius is $r_{d} \propto\left(J_{d}^{2} / M_{d}\right)$, increasing as the disk mass decreases.

For general studies of the evolution of an viscous accretion disk, the physics responsible for the removal of energy and transport of angular momentum is subsumed within the parameter $\alpha$ which fixes the viscosity $\nu$ driving the accretion. Using the typical parameterization $\nu=\alpha c_{s} H$ (Pringle \& Rees 1972; Shakura \& Sunyaev 1973) where $c_{s}$ is the local sound speed within the disk and $H=r c_{s} /\left(G M_{*} / r\right)^{1 / 2}$ is the local scale height of the disk, $\nu \propto r$ if the disk central temperature profile follows $T \propto r^{-1 / 2}$. Given the viscous timescale $t_{\nu}=r^{2} / \nu$, the viscous evolutionary time grows as $t_{\nu} \propto r$;

$$
t_{\nu} \simeq 10^{5} \mathrm{yr}\left(\frac{\alpha}{0.01}\right)^{-1}\left(\frac{r}{10 \mathrm{AU}}\right) .
$$

The viscous evolutionary timescale is plotted in Fig. 4 for two values of $\alpha\left(10^{-3}\right.$ and $10^{-2}$ ) which match best the observational accretion rate data for young stars (Hartmann et al. 1998). The viscous time at the edge of the disk sets the rate at which the disk expands; thus, $r_{d} \propto t_{\nu}$ and $M_{d} \propto t^{-1 / 2}$ for times greater than $t_{\nu 0}$, the initial viscous timescale. Setting the initial disk radius at $r_{d 0}$,

$$
\dot{M}_{\nu} \simeq 5 \times 10^{-7} M_{\odot} \mathrm{yr}^{-1}\left(\frac{\alpha}{0.01}\right)\left(\frac{r_{d 0}}{10 \mathrm{AU}}\right)^{-1}\left(\frac{t}{t_{\nu 0}}\right)^{-3 / 2} .
$$

Initially accretion onto the central star via viscous processes in the disk produces efficient disk dispersal; however, as the disk viscously spreads outward to satisfy the conservation of angular momentum, the accretion rate onto the central star decays and viscous accretion becomes a negligible mechanism for disk removal.

\section{Close Encounters with Nearby Stars}

When stars with disks have close encounters with other stars tidal torques are produced within the disk which may liberate disk material. Extensive studies by Clarke \& Pringle (1993), Heller (1995), Hall et al. (1996), Larwood (1997) and Bonnell \& Kroupa (1998) show that typically the disk is truncated to $1 / 3$ the impact parameter $r_{p}$ in a single close encounter. In an environment with a stellar number density $n_{*}$ and a velocity dispersion $v_{*}$ the timescale for disk truncation to $r_{d}$ is $t_{S E}=\left(n_{*} \sigma v_{*}\right)^{-1}$, where $\sigma=\pi\left(3 r_{d}\right)^{2}$ is the cross section for interaction. Substituting the typical number density and velocity dispersion of stars in the Trapezium region of Orion, the time required to truncate a disk to $r_{d}$ is

$$
t_{S E} \simeq 2 \times 10^{7} \mathrm{yr}\left(\frac{n_{*}}{10^{4} \mathrm{pc}^{-3}}\right)^{-1}\left(\frac{v_{*}}{1 \mathrm{~km} \mathrm{~s}^{-1}}\right)^{-1}\left(\frac{r_{d}}{100 \mathrm{AU}}\right)^{-2} .
$$


The timescale for disk dispersal via stellar encounters is plotted in Fig. 4. Only very large disks are truncated in reasonable times via this process and only in extremely populated regions such as the Orion Trapezium.

\section{Stellar Winds}

While observations show that young stars produce extremely powerful winds with mass-loss rates $\dot{M}_{w} \sim 10^{-7} \mathrm{M}_{\odot} \mathrm{yr}^{-1}$ and terminal velocities $V_{w} \sim 100-200$ $\mathrm{km} \mathrm{s}^{-1}$, these outflows are typically collimated away from the disk plane and are responsible for driving bipolar jets and outflows. The driving mechanism for these strong winds is believed to be the interaction between rigid rotating magnetic fields and accretion of material through the inner disk, although the exact structure and location of the magnetic fields is still strongly debated (Shu et al. 1988; Königl 1995; Ouyed \& Pudritz 1997a,b). As the accretion processes decay, magnetic activity in the chromosphere of the young, low mass star, drives a spherically symmetric stellar wind which is likely to remain much more active than the current stellar wind; thus, $\dot{M}_{w}>10^{-14} \mathrm{M}_{\odot} \mathrm{yr}^{-1}$ for $\sim 10^{7} \mathrm{yr}$.

Disk erosion via stellar wind dispersal has been postulated by Cameron (1973) and Horedt $(1978,1980)$. However, in these papers the bulk properties of the wind, such as total energy and momentum, were compared with bulk properties of the disk to determine the fate of disk material. Elmegreen (1979) considered the interface between the disk and the wind, determining that the turbulent layer produced would enhance viscosity and accretion through the disk.

An upper limit to the effectiveness of disk erosion via stellar winds can be estimated by considering the location of the boundary between the disk and wind. In steady state, the normal component of the ram pressure within the wind $(\sin \theta)^{2}\left(M_{w} V_{w} / 4 \pi r^{2}\right)$ must equal the local thermal support of the disk $\rho\left(r, z_{s}\right) c_{s}^{2}(r)$ along the interface (see Fig. 1). Assuming that disk material migrates through the interface at a small fraction of the local sound speed $\epsilon \sim 0.01-$ 0.1 (cf. Cantó \& Raga (1991) for the case of entrainment around jets) and is entrained within the wind, the mass loss rate can be approximated (Johnstone $\&$ Hollenbach 2000). Assuming a cold disk with $c_{s}=0.1\left(G M_{*} / r\right)^{1 / 2}$ :

$$
\dot{M} \simeq 4 \times 10^{-2} \dot{M}_{w}\left(\frac{V_{w}}{100 \mathrm{~km} \mathrm{~s}^{-1}}\right)\left(\frac{\epsilon \sin ^{2} \theta}{10^{-4}}\right)\left(\frac{T\left(r_{d}\right)}{100 \mathrm{~K}}\right)^{1 / 2}
$$

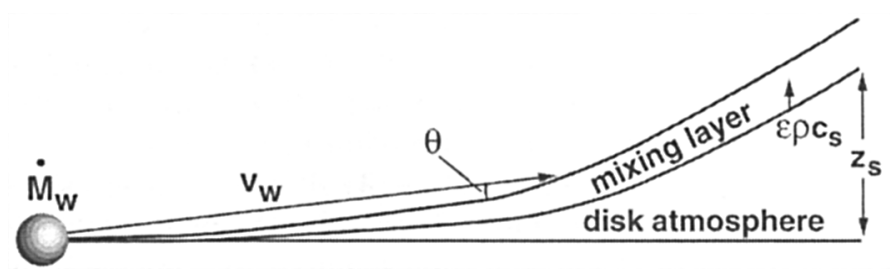

Figure 1. Schematic representation of the stripping of disk material due to the shear caused by the impact of a stellar wind. 
and

$$
t_{W S} \simeq 10^{7} \mathrm{yr}\left(\frac{r}{1 \mathrm{AU}}\right)^{1 / 4}\left(\frac{M_{d}}{0.01 \mathrm{M}_{\odot}}\right)\left(\frac{V_{w}}{100 \mathrm{~km} \mathrm{~s}^{-1}}\right)^{-1}\left(\frac{\dot{M}_{w}}{10^{-8} \mathrm{M}_{\odot} \mathrm{yr}^{-1}}\right)^{-1} .
$$

In the latter equation we have implicitly assumed the numerically computed $\epsilon \sin ^{2} \theta \sim 10^{-4}$ for a reference model with $\dot{M}_{w}=10^{-8} \mathrm{M}_{\odot} \mathrm{yr}^{-1}$ and $V_{w}=100$ $\mathrm{km} \mathrm{s}^{-1}$. The disk dispersal timescale due to stellar winds is plotted in Fig. 4, showing that, except in the very inner disk, wind stripping is not a significant disk dispersal mechanism.

\section{Photoevaporation of Disks}

An efficient mechanism for the removal of disks is photoevaporation via ultraviolet photon heating. Given a strong flux of ultraviolet photons, the disk surface is heated to $10^{3}-10^{4} \mathrm{~K}$ depending on the hardness of the radiation field, and over most of the disk the material becomes unbound producing a slow 3-10 km $\mathrm{s}^{-1}$ thermal disk wind. Absorption and scattering of the ultraviolet radiation field through the wind limits the effectiveness of the evaporation and produces an equilibrium between heating at the disk surface and mass-loaded flow.

\subsection{Photoevaporation by the central star}

If the embedded star, at the center of the disk, is responsible for the ultraviolet photon flux than photons must be able to penetrate through the inner regions of the disk, where heating the disk surface is not sufficient to launch a disk wind but rather creates a thick bound corona, to reach the outer regions from which the thermal disk wind launches. Hollenbach et al. (1994) showed that for extreme ultraviolet photons, which heat the disk surface to $10^{4} \mathrm{~K}$ through photoionization of hydrogen, recombinations in the hot disk corona produce an indirect ultraviolet radiation field which penetrates to great distances despite the rapid attenuation along the direct path from the star to the outer disk. Figure 2a shows a schematic of the radiative transfer and flow characteristics for such a case. A powerful radially directed wind (Fig. $2 b$ ) may also help reduce the attenuation produced in the inner regions of the disk by pushing out the hot disk corona.

Semi-analytic models for the destruction of disks via ionizing ultraviolet photons from the embedded star were first attempted for massive stars (Hollenbach, Johnstone, \& Shu 1993; Hollenbach et al. 1994) and provide a reasonable explanation for the observed radio size, emission measure, and lifetimes for many of the enigmatic ultracompact HII regions cataloged initially by Wood \& Churchwell (1989). The photoevaporation models were extended to cover the dispersal of possible protoplanetary disks around young, low mass stars by Shu, Johnstone, \& Hollenbach (1993). Numerical simulations have been performed by Yorke \& Welz $(1993,1994)$ and Richling \& Yorke $(1997,1998)$ and are in significant agreement with the analytic results.

The detailed solution of the semi-analytic work requires knowledge of the number of ionizing photons emitted by the central star $\phi_{i}$, as these models only considered extreme ultraviolet heating via photoionization, and the location 
(a) Weak Stellar Wind

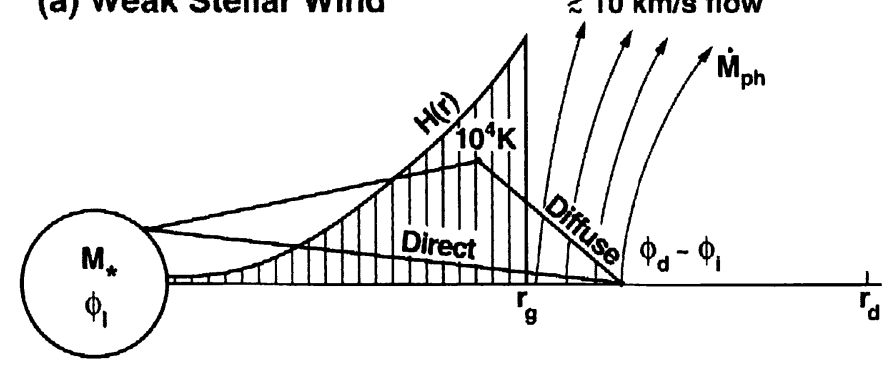

\section{(b) Strong Stellar Wind}

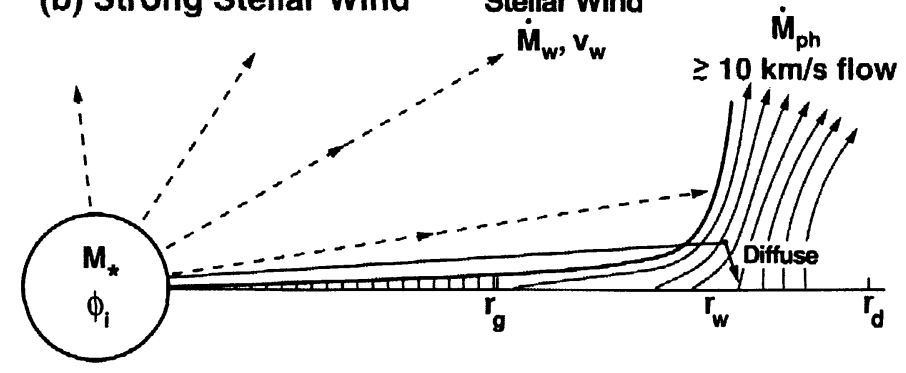

Figure 2. Schematic representation of photoevaporation by the central star. On the top (a) is the case with insignificant stellar wind. On the bottom (b) is the strong wind case.

within the disk $r_{g}$ where the thermal disk wind begins. Equating the sound speed in the ionized gas $c_{I I}$ with the escape velocity at $r_{g}$ yields $r_{g}=G M_{*} / c_{I I}^{2}$. For a $1 \mathrm{M}_{\odot}$ star $r_{g} \simeq 9 \mathrm{AU}$. The mass-loss rate in steady state is

$$
\dot{M}_{c} \simeq 4 \times 10^{-10} \mathrm{M}_{\odot} \mathrm{yr}^{-1}\left(\frac{\phi_{i}}{10^{41} \mathrm{~s}^{-1}}\right)^{1 / 2}\left(\frac{M_{*}}{\mathrm{M}_{\odot}}\right)^{1 / 2}
$$

for the case of a weak stellar wind. For a strong stellar wind the mass-loss is

$$
\dot{M}_{c} \simeq 4 \times 10^{-10} \mathrm{M}_{\odot} \mathrm{yr}^{-1}\left(\frac{\dot{M}_{w}}{10^{-10} \mathrm{M}_{\odot} \mathrm{yr}^{-1}}\right)\left(\frac{V_{w}}{100 \mathrm{~km} \mathrm{~s}^{-1}}\right),
$$

although the exact mass-loss rate in the strong wind case depends sensitively on the size of the disk. In the strong wind case the mass-loss rate per unit surface area $\propto r^{-3 / 2}$ over much of the disk and assuming that the surface density distribution of the disk follows $\Sigma \propto r^{-3 / 2}$ (Hayashi et al. 1985), the timescale for evaporation at $r>r_{g}$ is

$$
t_{c}(\operatorname{evap}) \simeq 10^{7} \mathrm{yr}\left(\frac{\phi_{i}}{10^{41} \mathrm{~s}^{-1}}\right)^{-1 / 2}\left(\frac{\Sigma_{0}}{\Sigma_{0}(\min )}\right)
$$



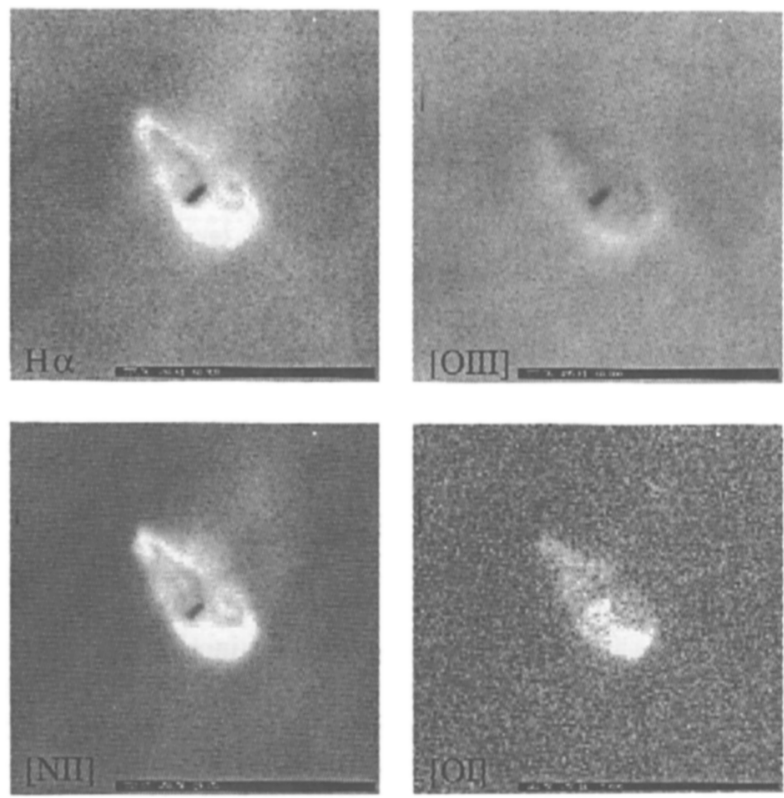

Figure 3. HST images of HST 182-413 (HST 10) in H $\alpha$, [O III], [S II], and [OI] $6300 \AA$. The disk appears in absorption for most optical lines except [O I]. The ionization front, seen clearly in $\mathrm{H} \alpha$ is offset from the disk surface.

where $\Sigma_{0}$ is the disk surface density at a fiducial radius and $\Sigma_{0}(\min )$ is the corresponding value for the minimum solar nebula $\left(M_{d} \simeq 0.01 \mathrm{M}_{\odot}\right)$. Figure 4 plots the disk dispersal timescale due to photoevaporation by the central star against the disk dispersal timescales via other processes.

\subsection{Photoevaporation by external sources}

Low mass stars born in clusters may be subjected to intense ultraviolet radiation fields produced by the nearby massive stars. The 'proplyds' in the Orion Nebula (Fig. 3) are the best examples of star-disk systems undergoing photoevaporation and photoionization of disk material due to the proximity of $\mathrm{O}$ stars (Bally et al. 1998; O'Dell 1998 and references therein).

For external photoevaporation the illumination of the disk does not require complicated radiative transfer, rather the photons need to travel upstream through the spherically divergent disk wind flow. Thus, to balance the ionizing photon flux $F_{i}=\phi_{i} /\left(4 \pi d^{2}\right)$, where $d$ is the distance between the low mass star disk system and the source of ionizing photons, with recombination requires a base density $n_{0} \simeq\left(3 F_{i} /\left(\alpha_{r} r_{i}\right)\right)^{1 / 2}$, where $\alpha_{r}$ is the recombination coefficient for $10^{4} \mathrm{~K}$ ionized gas and $r_{i}$ is the location of the ionization front (Johnstone, Hollenbach, \& Bally 1998; Störzer \& Hollenbach 1999). If the ionization front is 


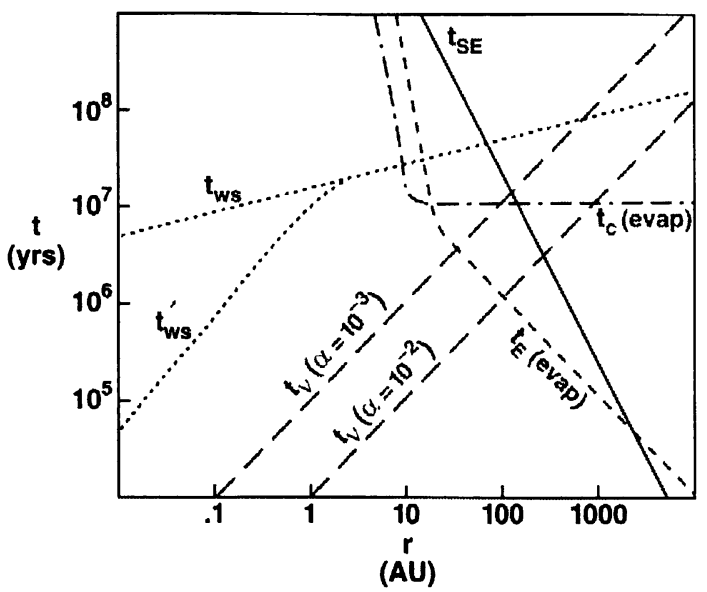

Figure 4. Timescales for disk dispersal: $t_{\nu}$ is the viscous timescale for $\alpha=10^{-2}$ and $10^{-3}(\S 3) ; t_{S E}$ is the stellar encounter (tidal stripping) timescale for Trapezium cluster conditions $(\S 4) ; t_{w s}$ and $t_{w s}^{\prime}$ are stellar wind stripping timescales for wind and disk parameters summarized in $\S 5 ; t_{c}$ (evap) is the photoevaporation timescale by the central star (strong wind case) and $t_{E}$ (evap) is the photoevaporation timescale for an external star (Trapezium conditions) for the conditions summarized in $\S 6$.

coincident with the disk then the mass loss rate is $\dot{M}_{e} \propto \phi_{i}^{1 / 2} d^{-1} r_{d}^{3 / 2}$ :

$$
\dot{M}_{e}^{E U V} \simeq 7 \times 10^{-9} M_{\odot} \mathrm{yr}^{-1}\left(\frac{\phi_{i}}{10^{49} \mathrm{~s}^{-1}}\right)^{1 / 2}\left(\frac{r_{d}}{10 \mathrm{AU}}\right)^{3 / 2}\left(\frac{10^{17} \mathrm{~cm}}{d}\right) .
$$

Johnstone et al. (1998) showed that in general the far ultraviolet photons (FUV), which heat the molecular and neutral gas to $\sim 10^{3} \mathrm{~K}$, are most efficient at liberating material from around young, externally evaporated disks. In this case the column of material within the flow regulates the attenuation of FUV photons toward the disk and equilibrium is expected when $\tau_{\text {FUV }} \sim 1$ requiring a column $N_{D} \sim 10^{21} \mathrm{~cm}^{-2}$. Thus, $n_{0} \simeq r_{d} / N_{D}$ and $\dot{M}_{e} \propto r_{d}$ :

$$
\dot{M}_{e}^{F U V} \simeq 2 \times 10^{-8} \mathrm{M}_{\odot} \mathrm{yr}^{-1}\left(\frac{N_{D}}{5 \times 10^{21} \mathrm{~cm}^{-2}}\right)\left(\frac{r_{d}}{10 \mathrm{AU}}\right) .
$$

Whenever the mass loss due to FUV photons dominates over the mass loss due to ionizing photons the ionization front moves away from the disk, since the ionizing photon flux cannot penetrate through the dense wind. This phenomenon is observed in Fig. 3.

External evaporation most efficiently removes the outer edge of the disk and thus the erosion process truncates the disk over time. Figure 4 plots the time to truncate the disk to $r_{d}$. External evaporation is most efficient for large 
disks, where the surface area suffering erosion is highest and the surface densities lowest, and becomes a considerably slower process once the disk has become highly truncated. Eventually, the disk becomes small enough that the photon heating is unable to produce a flow $r_{d}<r_{g}$. Details on the evolution of externally evaporated disks and analysis of the disk properties of the Orion proplyds are contained in Johnstone et al. (1998) and Störzer \& Hollenbach (1999).

\section{Conclusions}

Figure 4 plots a summary of the possible disk truncation timescales. Destroying the entire disk on timescales of $10^{7} \mathrm{yr}$ requires more than one disk destruction process. Perhaps the most promising scenario is the combination of external evaporation and viscous accretion. As the disk is truncated to smaller radii the evaporation and viscous evolutionary times converge. At the location where the two timescales are identical one would expect the disk to evaporate efficiently (and on that timescale) as viscous spreading continually moves new material toward the disk edge and evaporation removes the material from the disk. Thus, stars born in hostile environments such as the Orion Nebula should experience disk dispersal on timescales of $10^{6}-10^{7} \mathrm{yr}$.

Acknowledgments. I would like to thank my colleagues David Hollenbach, Harold Yorke, Frank Shu, and John Bally for many interesting discussions on the importance of disk dispersal around young stars. This work was supported by the Natural Sciences and Engineering Research Council of Canada and the Canadian Institute for Theoretical Astrophysics.

\section{References}

Adams, F.C., Emerson, J.P., \& Fuller, G.A. 1990, ApJ, 357, 60

Bally, J., Sutherland, R.S., Devine, D., \& Johnstone, D. 1998, AJ, 116, 293

Beckwith, S.V.W., Sargent, A.I., Chini, R.S., \& Guesten, R. 1990, AJ, 99, 924

Bodenheimer, P. \& Pollack, J.B. 1986, Icarus, 67, 39

Bonnell, I. \& Kroupa, P. 1998, in The Orion Complex Revisited, eds. M.J. McCaughrean \& A. Burkert (ASP), in press

Cameron, A.G.W. 1973, Icarus, 18, 407 1995, Meteoritics, 30, 133

Cantó, J. \& Raga, A. 1991, ApJ, 372, 646

Clarke, C.J. \& Pringle, J.E. 1993, MNRAS, 261, 190

Elmegreen, B.G. 1979, A\&A, 80, 77

Greenberg, R., Hartmann, W.K., Chapman, C.R., \& Wacker, J.F. 1978, Icarus, 35,1

Greenberg, R., Weidenschilling, S., Chapman, C.R., \& Davis, D.R. 1984, Icarus, 59,87

Hall, S.M., Clarke, C.J., \& Pringle, J.E. 1996, MNRAS, 278, 303

Hartmann, L., Calvet, N., Gullbring, \& E., D'Alessio, P. 1998, ApJ, 495, 385

Hayashi, C., Nakazawa, K., \& Nakagawa, Y. 1985, in Protostars and Planets II (U. Ariz. Press), 1100

Heller, C.H. 1995, ApJ, 455, 252 
Hollenbach, D., Johnstone, D., \& Shu, F. 1993, in Massive Stars: Their Lives in the Interstellar Medium, eds. J.P. Cassinelli \& E.B. Churchwell, ASPCS 35, 26

Hollenbach, D., Johnstone, D., Lizano, S., \& Shu, F. 1994, ApJ, 428, 654

Hollenbach, D.J., Yorke, H.W., \& Johnstone, D. 1999 in Protostars and Planets IV, eds. V.G. Mannings et al. (Univ. Ariz.), in press.

Horedt, G.P. 1978, A\&A, 64, 173 1980, A\&A, 92, 267

Johnstone, D. \& Hollenbach, D.J. 2000, in preparation

Johnstone, D., Hollenbach, D., \& Bally, J. 1998, ApJ, 499, 758

Königl, A. 1995, Rev. Mex. AC, 1, 275

Larwood, J.D. 1997, MNRAS, 290, 490

Laughlin, G. \& Bodenheimer P. 1994, ApJ, 436, 33

Lin, D.N.C. \& Papaloizou, J. 1985, in Protostars \& Planets II (Univ. Ariz.), 981 1986a, ApJ, 307, 395 $1986 \mathrm{~b}, \mathrm{ApJ}, 309,846$

Lissauer, J.J. 1987, Icarus, 69, 249

1993, ARAA, 31, 129

Marcy, G.W., Cochran, W.D., \& Mayor, M. 1999, in Protostars and Planets IV, eds. V.G. Mannings et al. (Univ. Ariz.), in press

Mizuno, H. 1980, Prog. Theor. Phys., 64, 544

Nakagawa, Y., Hayashi, C., \& Nakazawa, K. 1983, Icarus, 54, 361

O'Dell, C.R. 1998, AJ, 115, 263

Ouyed, R. \& Pudritz, R. 1997a, ApJ, 482, 712 1997b, ApJ, 484, 794

Papaloizou, J. \& Lin, D.N.C. 1984, ApJ, 285, 818

Pollack, J.B., Hubickyj, O., Bodenheimer P., Lissauer J.J., Podolak M., \& Greenzweig Y. 1996, Icarus, 124, 62

Pringle, J.E. \& Rees, M.J. 1972, A\&A 21, 1

Richling, S. \& Yorke, H.W. 1997, A\&A, 327, 317 1998, A\&A, 340, 508

Safronov, V.S. 1969, Evolution of the protoplanetary cloud and formation of Earth and the planets (Nauka, Moscow)

Shakura, N.I. \& Sunyaev, R.A. 1973, A\&A, 24, 337

Shu, F.H., Johnstone, D., \& Hollenbach, D. 1993, Icarus, 106, 92

Shu, F.H., Lizano, S., Ruden, S.P., \& Najita, J. 1988, ApJ, 328, L19

Störzer, H. \& Hollenbach, D. 1999, ApJ, 515, 669

Terebey, S., Shu, F.H., \& Cassen, P. 1984, ApJ, 286, 529

Wood, D.O.S. \& Churchwell, E. 1989, ApJS, 69, 831

Weidenschilling, S.J. 1984, Icarus, 60, 553

Wetherill, G.W. 1980, ARAA, 18, 77

Wetherill, G.W. \& Stewart, G.R. 1986, L\&PS 17, 939

Yorke H.W., Bodenheimer P., \& Laughlin, G. 1995, ApJ, 443, 199

Yorke, H.W. \& Welz, A. 1993, in Star Formation, Galaxies and the Interstellar Medium, eds. J. Franco et al. (Cambridge U. Press), 239 1994, in Numerical Simulations in Astrophysics, eds. J. Franco et al. (Cambridge U. Press), 318 


\section{Discussion}

M. G. Burton: Does the [OI] $6300 \AA$ emission come from the dense PDR (and hot FUV field) or from the edge of the H II region? It can get very hot (several $1000 \mathrm{~K}$ ) at the edge of such PDRs where O I can be collisionally excited.

D. Johnstone: The [OI] emission we are trying to explain occurs not at the outer edge of the PDR (at the ionization front) but rather at the base of the PDR front down on the disk surface. In fact the base of the disk glows bright in $[\mathrm{OI}]$, then there is virtually no [O I] emission until the ionization front. Thus the FUV field is not responsible for directly producing [O I] $6300 \AA$ emission at the disk base and we need to invoke chemistry.

A. Richards: How big would a dust particle need to be to survive in the disk and not be blown away?

D. Johnstone: We have assumed that typical dust particles would be entrained in the flow off the disk but I have not done a detailed calculation to determine the maximum size that could be lifted. Given the number density of hydrogen $\sim 10^{6} \mathrm{~cm}^{-3}$ and velocity $3-10 \mathrm{~km} \mathrm{~s}^{-1}$ we should have no problem with typical dust and there may be a better coupling due to charge effects.

$D$. W. Koerner: What do you mean by viscous dispersal time if, as you have said, much of the material is never lost from the system?

D. Johnstone: The removal of material goes both out and in. Material not accreted on the star is moved further out where it is more susceptible to dispersal by other means. 


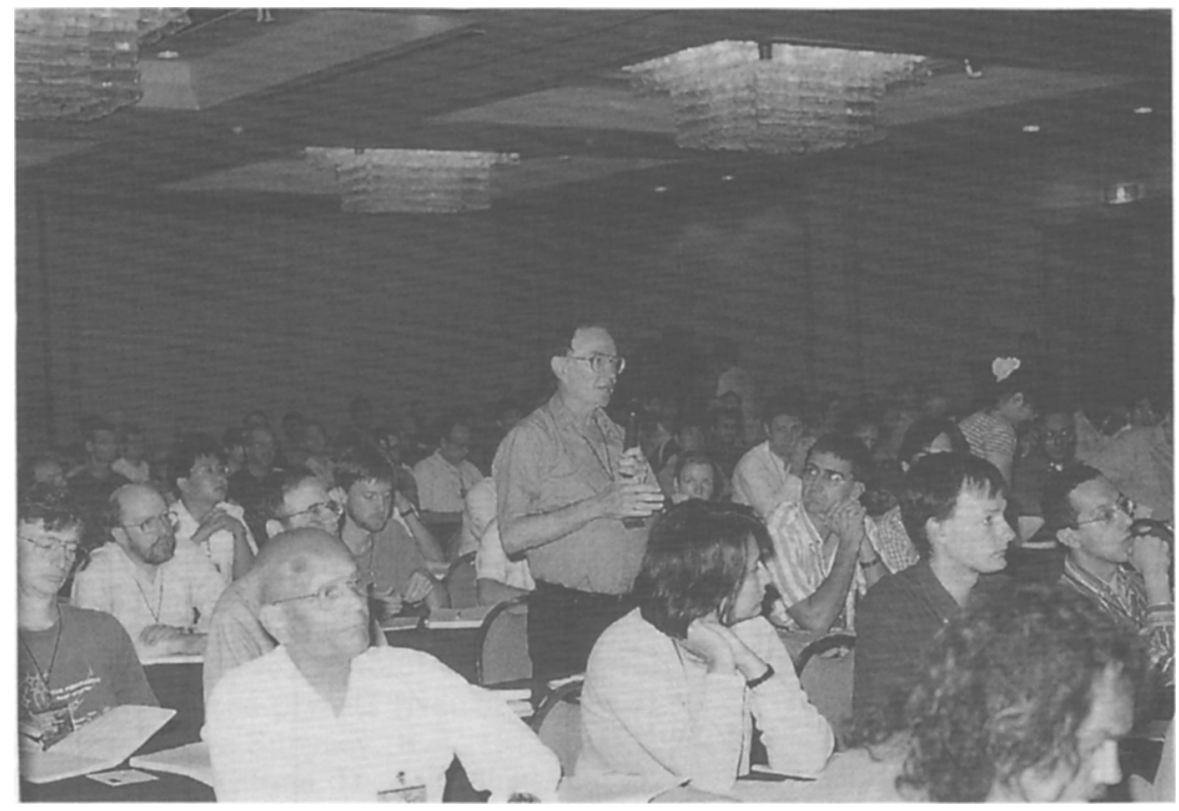

